\title{
Research on the method of
}

\section{assembly constraints products compact layout}

\author{
Junyan Ma ${ }^{1, a}$, Zhi Guo ${ }^{2, b}$, Xiaoping Liao ${ }^{3, \mathrm{c}}$ and Xiangsha Liu ${ }^{4, \mathrm{~d}}$ \\ ${ }^{1}$ Mechanical manufacturing \& automation, science and technology department, \\ Guangxi University, Nanning, Guangxi, China \\ ${ }^{2}$ Mechanical manufacturing \& automation, science and technology department, \\ Guangxi University, Nanning, Guangxi, China \\ ${ }^{3}$ Mechanical manufacturing \& automation, science and technology department, \\ Guangxi University, Nanning, Guangxi, China \\ ${ }^{4}$ Mechanical manufacturing \& automation, science and technology department, \\ Guangxi University, Nanning, Guangxi, China

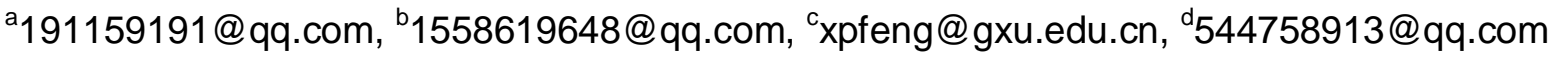

Keywords: compact layout convex hull plus rubber band assembly constraint

Abstract. We put forward the compact convex hull plus rubber band layout model based on the engineering practical problems. Under the assembly constraints the assembly constraints layout model is set up. We can get the exterior outline feature of the layout objects through Graph Outline Recognition Technology. Under the elastic force of a rubber band to get the initial layout. Three layout adjustment strategies are proposed to get the better layout results in the end. We set an example about engine compartment layout problem to illustrate the feasibility of the method.

\section{Introduction}

Layout problems explore that several objects are placed in a limited space under various constraints to make optimal indicators. Layout problems are widely used in engineering practice. For example, container lading problems[1], integrated circuit layout problem[2], engine compartment layout problem[3] and so on. To solve the difficult layout problem, Kim Y[4] put forward a a rule-based heuristic algorithm to solve the problem of automatic layout arrangement. Hasimshony[5] used graphic-theory to bulid layout model, which was to solve the layout prolblem with constraints. Sum put forward an ant colony optimization based layout optimization algorithm to solve some related optimization problems. This paper is to solve two-dimensional layout problem under the condition of the assembly on the basis of convex hull plus rubber model.

\section{Compact layout condition model in the assemble}

Three necessary conditions must be considered in the assembly.

(1) All the objects must be packed in the given space.

(2)There is no interference between two objects $Q_{i j}\left(X_{i}\right)$. 
(3) Considering the cost of the assembly and disassembly, space assembly constraints set $\delta$ are introduced.

For a given set $Q=\left[Q_{1}, Q_{2}, \ldots, Q_{n}\right]$ of $n$ layout objects described as polygons and a layout space $C$. We can get the Eq. 1 .

$$
\begin{cases}\text { Find } & X=\left[x_{1}, x_{2}, \ldots, x_{k}, \ldots, x_{n}\right]^{T} \in R^{N} \\ \max & \varepsilon(X) \\ \text { s.t. } & X_{i} \subset C, \quad X_{i}=X_{i}+\delta \\ & \sum_{i=1}^{n} \sum_{j=1}^{i=1} O_{i j}\left(X_{i}\right)+\sum_{i=1}^{n} C_{i}\left(X_{i}\right)=0\end{cases}
$$

Compactness $\varepsilon$ is one of the most important indicators to be pursued during the layout which is represented $S(r u b)$. Here are the two areas, one is the total of the objects, another is the surrounded by the rubber band. The ration of the two areas which is called compactness. We represent the area of each object is $S\left(o b j_{i}\right)$. We can get the Eq. 2 .

$$
\varepsilon=\frac{\sum_{i=1}^{n} S\left(o b j_{i}\right)}{S(r u b)}
$$

\section{Solution to the compact layout in the assembly}

We should simplify the layout model to improve the efficiency of the work of the computer, including two aspects.

On the one hand, we need to simplify all the layout objects. We only need to get the appearance of the layout component characteristics because we don't consider the component internal details. We can use Graph Outline Recognition Technology[6] to get the exterior outline feature of the layout objects. The manual mode for component that is shown in Fig. 1, we can use Graph Outline Recognition Technology to get the component of external outline that is shown in Fig. 2.

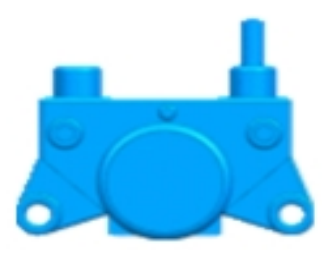

Fig. 1 Manual mode for component

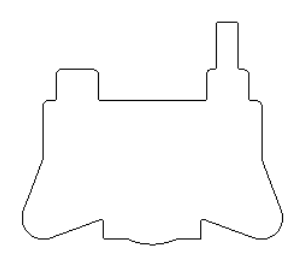

Fig. 2 Component of external outline

On the other hand, we should get simulated rubber band to form a convex hull plus rubber band layout model. We can discrete layout objects as a set point, then we seek minimum convex hull of the set point[7] as is shown in Fig. 3.

Under the force of rubber band, the initial layout objects occur translational motion, rotational motion or planar motion until the end. We put forward in the process of layout adjustment strategy because we can't ensure the layout of the result is the best. The following are three adjusting strategies. 
(1) Key layout object priority

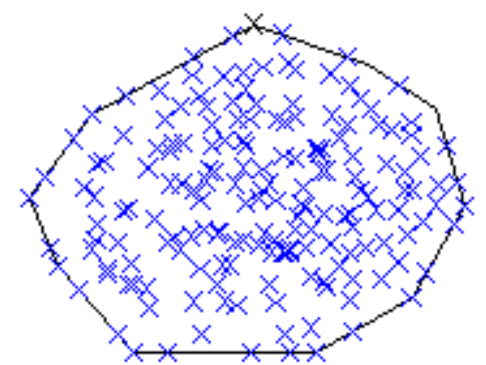

Fig. 3 Convex hull

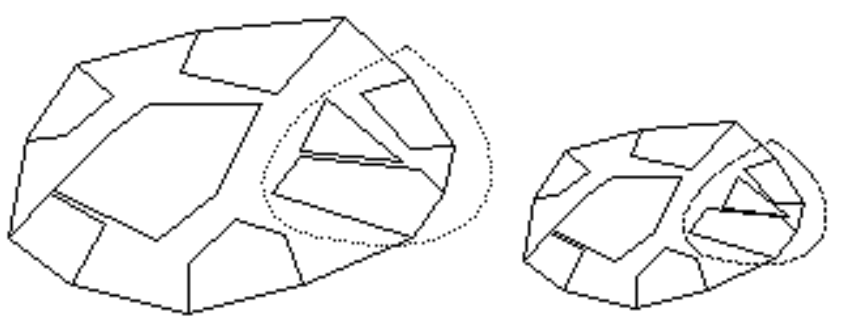

Fig. 4 Diagram of adjacency strategy

We define the maximum weight coefficient $W$ as the key layout object. Given priority to the objects of the bigger weight coefficient in the layout system, the rest are randomly generated in computer.

Algorithm of key layout object priority:

Step 1: Inputting $W[1,2,3, \ldots, \mathrm{n}]$.

Step 2: Comparing weight coefficient, according to from big go to small of in proper order arrange.

Step 3: Choosing the maximum weight coefficient $W$ as the key layout object. We gtve priority to the position of the key layout object.

Step 4: Determining the coordinates of each objetc in the layout system, and fixed their coordinates.

(2) Adjacency strategy

When the adjacent two objects are together in the motion under the force of the rubber band in the simulation layout, we can define the two objects are similar objects, which is shown in Fig. 4.

Algorithm of adjacency strategy:

Step 1: Inputting layout objects into the layout system.

Step 2: Setting time $t$.

Step 3: Determining whether two objects are similar objects. If they are, we can see them as a whole part to be participated in motion.

Step 4: Calculating the compactness of the layout system.

(3) Cancellation strategy

The layout system fall into a state of "stuck" when the layout objects moves to a moment in the process of layout, we can use cancellation strategy. Cancellation strategy has two kings of forms. When layout can't continue or occur a disadvantage, we can withdraw to the last step, and we use other strategies to relocate.

\section{Experiment}

Here are the main parts in automobile engine compartment in Fig. 5.

To solve the layout problem, we establish the flow chart in Fig. 6. We use Graph Outline Recognition Technology to get the outline of layout objects. And then, we optimize outline under the condition of the assembly. We get the compactness $\varepsilon=0.8873$ through VC++ software in the end. Accordingly, we get the final layout chart as is shown in Fig.7.

From the result, the compactness is greater than 0.85 , which means good result has been achieved. It proves that the convex hull of assembly constraint plus rubber band layout method is feasible. Likewise, this result provides a method for the engine of the layout design for automotive engine compartment layout design. 


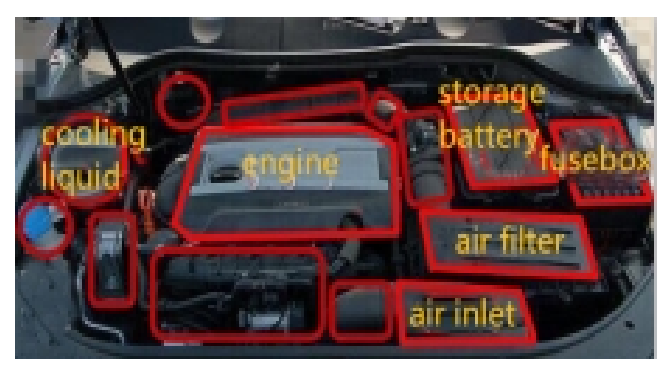

Fig. 5 Layout of engine compartment

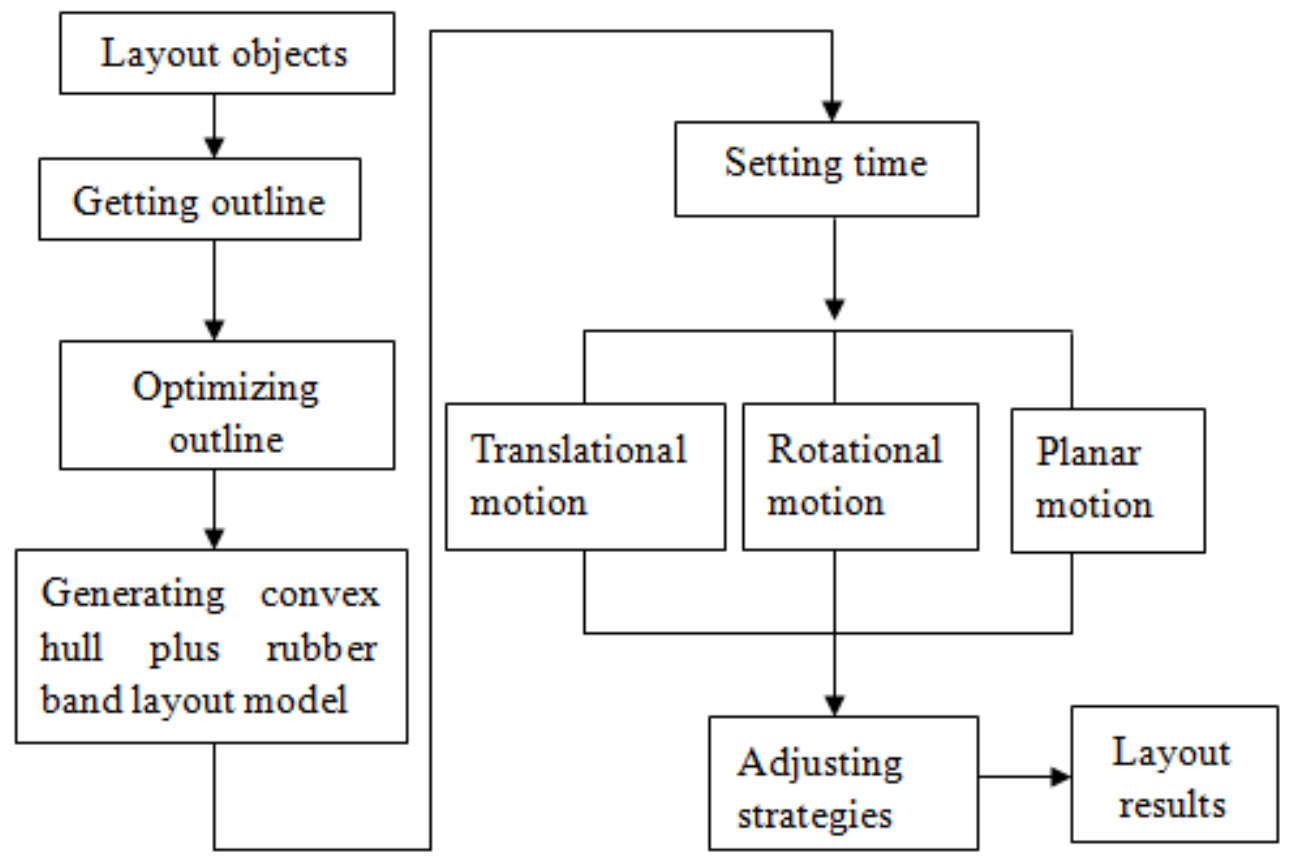

Fig. 6 Flow chart

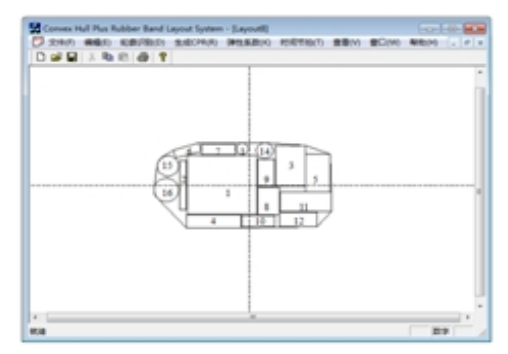

Fig. 7 Layout results

\section{Conclusion}

This article put forward the issue of the layout of the assembly constraints on the basis of the convex hull plus rubber band layout model. We give a simple example of the layout design for automobile engine compartment to show that the method is feasible and effective. 


\section{Acknowledgements}

We sincerely acknowledge the support of National Nature Science Foundation of China (51265002). Key Laboratory of Manufacturing System \& Advance Manufacturing Technology of GuangXi Province(12-071-11S04).

\section{References}

[1] Lee B K, Kim K H. Optimizing the yard layout in container terminals[J]. OR spectrum, 2013, 35(2): 363-398.

[2] Yijie Zeng. Layout method research of analogue integrated circuit[D]. Tsinghua University, 2004. In Chinese.

[3] Hong Dong, Paolo Guarneri, Georges Fadel. Bi-level Approach to Vehicle Component Layout With Shape Morphing[J]. Journal of Mechanical Design. 2011, Vol. 133 / 041008-1.

[4] Kim J Y. Graph theoretic heuristics for unequal-sized facility layout problems[J]. Omega, 1995, 23(4):391-401.

[5] Hashimshony R,Roth J.ALG: a model for generating alternative layout graphs under architectural constraints. Computer Aided Design,1986,18(8):431-43.

[6] Weiyong $\mathrm{Wu}$, Yinghui Wang, Yan Zhou. The research and rapid implementation of planar contour recognition and segmentation technique[J]. Journal of Graphics, 2003,24(4):14-19. In Chinese.

[7] Peide Zhou. Computer geometry, algorithm analysis and design[M]. Beijing: Tsinghua University Press, 2000. In Chinese. 